
4 5 6

\title{
EIder: A Compound Identification Tool for Gas Chromatography
}

\section{Mass Spectrometry Data}

\author{
Imhoi Koo, ${ }^{1,2}$ Seongho Kim, ${ }^{3}$ Biyun Shi, ${ }^{1,2}$ Pawel Lorkiewicz, ${ }^{4,5}$ Ming Song, ${ }^{6}$ Craig \\ McClain, ${ }^{5,6,7,8}$ and Xiang Zhang ${ }^{1,2,7, *}$
}

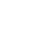

${ }^{1}$ Departments of Chemistry, ${ }^{6}$ Medicine, ${ }^{7}$ Pharmacology and Toxicology, ${ }^{2}$ Center for Regulatory and Environmental Analytical Metabolomics, ${ }^{4}$ Institute of Molecular Cardiology, ${ }^{5}$ Diabetes and Obesity Center, University of Louisville, Louisville, KY 40292, USA.

${ }^{3}$ Biostatistics Core, Karmanos Cancer Institute, Department of Oncology, School of Medicine, Wayne State University, Detroit, MI 48201, USA.

${ }^{8}$ Robley Rex Louisville VAMC, Louisville, Kentucky 40292, USA

\section{Corresponding Author}

Xiang Zhang, (phone) +01 502852 8878; (fax) +01 502 852-8149; (email)

xiang.zhang@louisville.edu. 


\section{Abstract}

22 We report software entitled EIder (EI mass spectrum identifier) that provides users with eight 23 literature reported spectrum matching algorithms for compound identification from gas

24 chromatography mass spectrometry (GC-MS) data. EIder calculates retention index according to 25 experimental conditions categorized by column class, column type and data type, where 9 26 empirical distribution functions of the absolute retention index deviation to its mean value were 27 constructed using the National Institute of Standards and Technology (NIST) 2011 retention 28 index database to improve the accuracy of compound identification. EIder filters compound 29 candidates based on elementary composition and derivatization reagent, and automatically adds 30 the molecular information of the native compound to each derivatized compound using a 31 manually created database. When multiple samples are analyzed together, EIder performs cross32 sample alignment and provides an option of using an average mass spectrum for compound 33 identification. Furthermore, a suite of graphical user interfaces are implemented in EIder to allow 34 users to both manually and automatically modify the identification results using experimental 35 information at various analysis stages. Analysis of three types of GC-MS datasets indicates that 36 the developed EIder software can improve the accuracy of compound identification.

37

38 KEY WORDS: Compound identification; GC-MS; Metabolomics; Retention index. 


\section{Introduction}

Gas chromatography-mass spectrometry (GC-MS) is one of the most widely used analytical

42 platforms for compound identification and quantification. A GC-MS instrument is usually

43 equipped with electron ionization (EI) ion source, and therefore provides retention time and EI

44 mass spectrum information for each compound. Compound identification is usually achieved by

45 matching an experimental EI mass spectrum to EI mass spectra recorded in a reference library

46 [1]. Retention indices (i.e., normalized retention times) can also be incorporated into

47 identification to reduce the rate of false identifications [2].

48 Various algorithms have been developed to improve the accuracy of mass spectrum 49 matching-based compound identification. Stein and Scott [1] proposed a composite measure with 50 ratio and inner product. $L_{1}$ - and $L_{2}$ - norm approaches were also introduced to identify chemical 51 compounds [3]. Koo, Zhang and Kim [4] developed Fourier and wavelet transform-based 52 composite (DFT/DWT) measures. Kim, Koo, Wei and Zhang [5] found the optimal weight 53 factors for $\mathrm{m} / \mathrm{z}$ value and intensity based on cosine correlation similarity. Kim, Koo, Jeong, Wu, 54 Shi and Zhang [6] also proposed mixture semi-partial and partial correlation-based measures. 55 Wei, Koo, Kim and Zhang [2] developed a method to simultaneously evaluate the mass spectrum 56 similarity and the retention index distance using an empirical mixture score function. Koo et al. 57 [7] demonstrated that each of these algorithms has limited identification accuracy and generates 58 different identification results. However, the software packages of these algorithms are not 59 publicly available for the research community to test and validate for further development.

60 GC-MS instrument vendors also developed several software packages for compound 61 identification, including Agilent ChemStation ${ }^{\circledR}$, Thermo Xcalibur $^{\circledR}$ and LECO ChromaTOF ${ }^{\circledR}$. 
62 These software packages are not open to the public and the details of the compound 63 identification algorithms are also not disclosed. In addition, none of these software packages 64 allows users to modify the identification results within the software.

65 The objective of this study was to develop a software package for compound identification 66 from the GC-MS data, where the users can use various analytical methods to modify the analysis 67 results before and after compound identification. We developed EIder (EI mass spectrum 68 identifier) using MATLAB 2010b. EIder performs mass spectrum matching using literature 69 reported eight spectrum similarity measures to improve the accuracy of compound identification. 70 It also enables users to manually and automatically modify the analysis results to reduce the false 71 identification rate using experimental information. The performance of EIder was tested by

72 analyzing three experimental datasets. One dataset was GC-MS data acquired from a mixture of 73 compound standards, while the other two datasets were GC-MS data of metabolite extracts from 74 mouse liver and feces. EIder and sample data can be accessed at 75 https://sourceforge.net/projects/metaopen/files/EIder/.

\section{2. Materials and methods}

\section{2.1. Experimental}

78 Three types of samples were prepared and analyzed on comprehensive two-dimensional gas 79 chromatography time-of-flight mass spectrometry (GC×GC-TOF MS) system, including a 80 mixture of compound standards, a metabolite extract from mouse liver, and 25 metabolite 81 extracts from mouse feces. The mixture of compound standards was a mixture of two 82 commercially available mixtures purchased form Sigma-Aldrich Corp. (Cat. No. A6407 and Cat. 
83 No. A6282, St. Louis, MO, USA) that contains 42 compounds each at $500 \mu \mathrm{M}$ except L-cystine 84 at $250 \mu \mathrm{M}$.

To extract metabolites from mouse liver and fecal samples, each sample was first weighed and homogenized on ice. The homogenized sample was added to ice cold $80 \%$ methanol with a ratio of $50 \mathrm{mg}$ of feces or liver in $1 \mathrm{~mL}$ of $80 \%$ methanol. The mixture was vortexed for $10 \mathrm{~min}$, followed by centrifugation for $10 \mathrm{~min}$ at $15000 \mathrm{rpm}$. Four-hundred microliters of the supernatant was aspirated into a glass vial and dried by SpeedVac overnight at $14{ }^{\circ} \mathrm{C}$. The dried metabolite extracts were dissolved in $40 \mu \mathrm{L}$ of the methoxyamine hydrochloride solution in pyridine (20 $\mathrm{mg} / \mathrm{mL}$ ) and vigorously vortex-mixed for $2 \mathrm{~min}$.

The metabolites in the mixture of metabolite standards were derivatized using N-methyl-Nwas dissolved in $40 \mu \mathrm{L}$ ethoxyamine hydrochloride pyridine solution $(30 \mathrm{mg} / \mathrm{mL})$ and vigorously

97 vortex-mixed for $1 \mathrm{~min}$. Methoxymation was carried out by sonicating for 20 min and incubating at $60{ }^{\circ} \mathrm{C}$ for 1 hour. Derivatization was conducted by adding $40 \mu \mathrm{L}$ of derivatization agent (MSTFA or MTBSTFA) to the glass vial and incubating at $60{ }^{\circ} \mathrm{C}$ for 1 hour. The stock solutions were then transferred to $\mathrm{GC}$ vials for analysis. The methoxymation and derivatization were prepared just before GC-MS analysis. All chemicals used for metabolite extract and derivatization were purchased form Sigma-Aldrich Corp. at St. Louis, MO, USA.

The LECO Pegasus 4D GC×GC-TOF MS instrument was equipped with an Agilent 6890 gas chromatograph and a Gerstel MPS2 auto-sampler (GERSTEL Inc., Linthicum, MD), featuring a 
$1060.25 \mathrm{~mm}^{1} d_{c} \times 0.25 \mu \mathrm{m}^{1} d_{f}$, DB-5ms GC capillary column (phenyl arylene polymer virtually 107 equivalent to a (5\%-phenyl)-methylpolysiloxane). A second GC column of $1 \mathrm{~m} \times 0.25 \mathrm{~mm}^{2} d_{c} \times$ $108 \quad 0.25 \mu \mathrm{m}^{2} d_{f}$, DB-17ms ((50\%-phenyl)-methylpolysiloxane) was placed inside the secondary GC 109 oven after the thermal modulator. Both columns were obtained from Agilent Technologies 110 (Agilent Technologies J\&W, Santa Clara, CA). The helium carrier gas (99.999\% purity) flow 111 rate was set to $2.0 \mathrm{~mL} / \mathrm{min}$ at a corrected constant flow via pressure ramps. The injector 112 temperature was set at $280{ }^{\circ} \mathrm{C}$. The primary column temperature was programmed with an initial 113 temperature of $60{ }^{\circ} \mathrm{C}$ for $0.5 \mathrm{~min}$ and then increased at $5^{\circ} \mathrm{C} / \mathrm{min}$ to $280{ }^{\circ} \mathrm{C}$ and kept for $15 \mathrm{~min}$. 114 The secondary column temperature program was set to an initial temperature of $70{ }^{\circ} \mathrm{C}$ for 0.5 115 min and then also increased using the same slope of temperature gradient employed in the first 116 column to $280{ }^{\circ} \mathrm{C}$. The thermal modulator was set to $+15^{\circ} \mathrm{C}$ relative to the secondary oven, and 117 a modulation time of $P_{M}=2 \mathrm{~s}$ was used. The mass range was set as $29-800 \mathrm{~m} / \mathrm{z}$ with an 118 acquisition rate of 200 mass spectra per second. The ion source chamber was set at $230{ }^{\circ} \mathrm{C}$ with 119 the transfer line temperature set to $280{ }^{\circ} \mathrm{C}$, and the detector voltage was $1450 \mathrm{~V}$ with electron 120 energy of $70 \mathrm{eV}$. The acceleration voltage was turned on after a solvent delay of $675 \mathrm{~s}$. The split 121 ratio was set at 20:1.

122 2.2. Analysis features of EIder

EIder has three main modules: a project meta-information module, a compound identification 124 module and an auxiliary display module (Figure 1). The project meta-information module is 125 designed mainly for loading input files and initializing parameters to be used for compound 126 identification. The compound identification module calculates mass spectral similarity scores 127 between query (experimental) spectra and reference spectra, using one or multiple spectral 128 similarity measures. The auxiliary display module enables users either manually or automatically 
129 to analyze data in order to reduce the rate of false identifications. These three analysis modules 130 are seamlessly integrated through multiple graphical user interfaces (Supplementary Figures S1131 S7).

\subsubsection{Input/output files and reference libraries}

133 The input files of EIder can be either raw GC-MS data in Netcdf (*.cdf) or XML (*.mzXML)

134 formats or a text-based comma-separated values (*.csv) generated from the raw GC-MS data 135 after spectrum deconvolution (Supplementary Table S1). Multiple files can be simultaneously 136 uploaded to EIder for batch analysis. The output file of EIder is a text file with comma-separated 137 values (*.csv), which is automatically saved in the same folder of the input files (Supplementary 138 Table S2).

139 EIder mass spectrum library contains all EI mass spectra available in public databases, 140 including MassBank [8] and NIST WebBook [9], where each compound is characterized by its

141 EI mass spectrum, compound name, chemical abstract system (CAS) number, molecular formula 142 and molecular weight. EIder also enables users to automatically build one or multiple user 143 reference databases by adding experimental mass spectra and related information into the EIder 144 mass spectrum library (Supplementary Figure S2). To enable users to use the commercial library, 145 EIder can extract reference EI mass spectra from NIST main library and its replicate library and 146 add them to the EIder library. The users are free to use one or a combination of multiple 147 databases in the EIder library for compound identification.

148 While a large reference library has a greater chance to contain a compound giving rise to the 149 experimental mass spectrum, the overall identification accuracy is reduced because there is a 150 greater chance that multiple reference spectra are similar to an experimental mass spectrum [7]. 151 To improve the accuracy of compound identification, EIder first allows users to remove the 
152 reference compounds from the EIder library based on compound elementary composition. All 153 compounds containing elements that are not specified by the users are excluded from spectrum 154 matching (Supplementary Figure S3 (d)). EIder further allows users to exclude compounds from 155 the reference library based on derivatization reagent used during experiment. Namely, any 156 compounds containing chemical groups from other derivatization reagents are removed from the 157 identification results. For example, any MSTFA derivatized compounds are removed from the 158 identification list if MTBASTFA was used to derivatize compounds (Supplementary Figure S3 $159(\mathrm{e}))$

\subsubsection{Spectrum matching algorithms}

A total of eight spectrum matching algorithms are implemented in EIder: Pearson's 162 correlation, $L_{1}$ - and $L_{2}$-norms [3], Stein and Scott's composite score [1], Fourier- and wavelet163 transform-based composite score [4], weighted cosine similarity with the optimal weight factor

164 [5], and semi-partial correlation mixture similarity scores [6] (see details in Supplementary 165 Section 1). One or multiple algorithms can be sequentially selected for compound identification. 166 The identification results of each chromatographic peak (using multiple identification 167 algorithms) are automatically organized for users to assess the identification precision. It has 168 been reported that the semi-partial correlation mixture similarity has the best identification 169 accuracy, but it is computationally expensive. The weighted cosine method is the fastest method 170 with comparable identification accuracy to the semi-partial correlation mixture similarity [7].

\section{$171 \quad 2.2 .3$ Retention index calculation}

172 Retention index has been widely used to assist compound identification. If the retention times 173 of a set of n-alkanes are available, EIder automatically calculates retention index such as Kovats 174 and Linear retention index for each compound based on experiment conditions. 
Kovats' retention index is defined as:

$$
I(x)=100 \times n+100\left(\frac{t_{R(x)}-t_{R(n)}}{t_{R(n+1)}-t_{R(n)}}\right)
$$

176 and linear retention index is defined as:

$$
I(x)=100 \times n+100\left(\frac{\log \left(t_{R(x)}^{\prime}\right)-\log \left(t_{R(n)}^{\prime}\right)}{\log \left(t_{R(n+1)}^{\prime}\right)-\log \left(t_{R(n)}^{\prime}\right)}\right)
$$

177 where $n, n+1$ are numbers of carbon atoms in two adjacent n-alkane standards, between

178 which a compound $x$ was eluted from the GC column, $t_{R}$ is a retention time and $t_{R}^{\prime}$ is adjusted 179 retention time.EIder performs retention index matching using our previously developed 180 algorithms [10]. Briefly, a total of 9 empirical distribution functions of the absolute retention 181 index deviation to its mean value were constructed using the NIST 2011 retention index database 182 information, owing to column class, column type and data type have significant effects on the 183 retention index values acquired on capillary columns. A retention index variation window is 184 determined from each distribution function using a user provided probability value.

\section{$185 \quad$ 2.2.4 Post identification analysis}

186 Compounds extracted from complex samples are often derivatized to make them volatile 187 and/or thermal stable for GC-MS analysis. A compound directly identified from a query 188 spectrum by EI mass spectrum matching is often the derivatized version of the compound 189 originally present in the sample. Therefore, each identified compound must be associated to its 190 underivatized form, i.e., the native form originally present in the sample. To this end, after 191 spectrum matching, EIder automatically adds the molecular information of the native compound 192 (name and CAS number) to each derivatized compound using our manually created database. 
After EI mass spectrum-based identification and estimation of retention index, EIder 194 generates an interactive plot of retention index vs. retention time of all identified compounds 195 (Figure 2), for users to further review and modify the identification results. EIder first 196 automatically fits the retention indices and retention times of all identified compounds into a 197 monotonic curve to show the global pattern of the retention indices of these identified 198 compounds corresponding to their retention times. The users can then easily recognize the 199 compounds with large distances of retention index to the fitted curve and manually remove these 200 compounds from the identification table. This process can be repeated until the users agree with 201 all identifications listed in the interactive plot.

202 EIder also automatically generates a suite of auxiliary tables and plots to assist users to 203 modify the identification results, including a hit list of a selected chromatographic peaks, 204 distribution of similarity scores for users to adjust the threshold of similarity scores, mass 205 spectral comparison plot(s) containing both reference and query mass spectra, and the spectral 206 similarity score distribution of top-50 hits of a selected chromatographic peak (Supplementary 207 Figures S5 and S6).

208 After identifying compounds, EIder further enables users to explore chemical information of 209 each identified compound by directly accessing multiple public databases, including PubChem, 210 ChemSpider and WebBook (Supplementary Figure S7).

\section{2. Results and Discussion}

The performance of spectrum similarity measures implemented in EIder has been previously 214 evaluated [6,7]. Therefore, we mainly focus on the usage of EIder's auxiliary functions for 215 purpose of improving the accuracy of compound identification. We evaluated the performance of 
216 EIder using three GC×GC-TOF MS datasets, respectively, acquired from a mixture of compound 217 standards, a metabolite extract from mouse liver, and 25 metabolite extracts from mouse feces. 218 Each experimental dataset was first processed using commercial software ChromaTOF to reduce 219 raw instrument data to a peak list, which has an EI mass spectrum, the first dimensional retention 220 time and the second dimension retention time for every detected chromatographic peak. The 221 deconvoluted information of each chromatographic peak was then used for compound 222 identification using both ChromaTOF and EIder, where the NIST 2011 library was used as 223 reference library.

224 While GC×GC-TOF MS data characterize each chromatographic peak by a mass spectrum 225 and two dimensional retention times, only the mass spectrum and the first dimension retention 226 time of each chromatographic peak were used in this study for compound identification. The 227 current version of EIder does not have the capability to calculate the retention index from the 228 second dimension retention time. In this study, the modulation period was set as $\mathrm{P}_{\mathrm{M}}=2 \mathrm{~s}$. 229 Compounds eluted from the first dimension column within a modulation period are mixed and 230 then subjected to the second dimension column for further separation, resulting in that these 231 compounds have the same first dimension retention time even though they may be well resolved 232 during the first dimension separation. Compared with the one dimension GC-MS, the retention 233 index converted from the first dimension retention time of GC×GC-TOF MS data contains a 234 certain degree of variation caused by modulation period. While all experiments were performed 235 on a GC $\times \mathrm{GC}-\mathrm{TOF}$ MS system in this study, the second dimension retention time of each 236 chromatographic peak was not used to test the performance of EIder. Therefore, the testing data 237 have the same information as the data acquired from one dimensional GC-MS, i.e., one 238 dimension retention times and mass spectra. 


\subsection{Analysis of data acquired from the mixture of compound standards}

240

241

242

243

244

245

246

247

248

249

250

251

252

253

254

255

256

257

258

259

260

261

Based on the company-provided information, the mixture of compound standards contains 42 compounds. After derivatization using MSTFA, the mixture was analyzed on GC $\times$ GC-TOF MS. The original peak list generated by ChromaTOF software consists of 243 chromatographic peaks. Figure S8 depicts a boxplot of similarity score distribution of the identified compound standards by ChromaTOF, weighted cosine and semi-partial correlation implemented in EIder, where the top hit (a compound with the highest spectrum similarity score) was considered as the identification result. It can be seen that the similarity scores of weighted cosine method and semi-partial correlation are higher than that of ChromaTOF, and the similarity scores of ChromaTOF have a much wider range than those of weight cosine and semi-partial correlation. By setting the threshold of spectral similarity $\geq 0.8$ in weighted cosine and semi-partial correlation and $\geq 800$ in ChromaTOF, the weighted cosine method implemented in EIder correctly identified 37 standard compounds while the semi-partial correlation in EIder identified 35 standard compounds and ChromaTOF only identified 31 standard compounds, respectively.

Table 1 lists five compounds that were the top hits for the EI mass spectra of five chromatographic peaks. Each of these was originally identified as an MTBSTFA-derivatized compounds. However, the sample was derivatized using MSTFA during the experiment and therefore, any identification results of MTBSTFA-derivatized compounds are false identifications. EIder automatically excludes all MTBSTFA-derivatized compounds using user provided derivatization method after spectrum matching. By doing so, the top hit for the chromatographic peak eluted at 26.65 min becomes "L-Proline, 1-(trimethylsilyl)-, trimethylsilyl ester" that is a MSTFA-derivatized compound and L-proline is a compound in the company provided list. 
EIder provides two options for users to apply retention indices to filter potential false 263 identifications. One is to calculate retention index using internal or external reference 264 compounds and then match the retention index with library information based on experiment 265 conditions [10]. The other is to use the interactive plot implemented in EIder. The users can 266 either select one of these two approaches or combine them together. Figure 2(a) depicts the 267 interactive plot that uses a regression curve to recognize compounds with large retention index 268 variations. The retention times of all compounds identified by mass spectrum matching were first 269 converted into retention indices. The pair of retention index and retention time of each 270 compound is then displayed in a scatter plot. The relation between retention indices and retention 271 times of all compounds is then fitted by a monotonic function. The users can then adjust the 272 confidence level of curve fitting to recognize false identifications. Any compounds with 273 retention indices outside of the confidence bands in regression analysis [11] (either above the 274 upper boundary curve or below the lower boundary curve in Figure 2(a)) are likely to be 275 potential false identifications. The user can examine details of these identifications using the 276 identification table displayed on the left side of the interactive plot. For example, the users can 277 further examine the chemical and biochemical information of an identified compound online by 278 navigating multiple public databases, including PubChem, ChemSpider and WebBook, by 279 clicking on an identified compound listed in the identification table. However, it should be noted 280 that the retention index information for many of the detected compounds is not available in the 281 current retention index library. For this reason, the interactive plot only shows the information 282 for compounds that have retention index data in the NIST WebBook library 283 (http://webbook.nist.gov/). 
A high spectrum similarity threshold for mass spectral matching can induce a high rate of false negative identifications, while a low spectral similarity threshold can introduce a high rate of false positive identifications. Currently, the threshold of spectral similarity is selected by users based on their experience and confidence on the GC-MS data. EIder recommends a threshold of spectral similarity by investigating the relationship of retention index deviations between the 289 reference values and experimental values of all identified compounds, as well as receiver 290 operating characteristic (ROC) curve [12]. An optimal threshold of the mass spectral similarity is 291 recommended as the spectral similarity that provides the largest true positive rate (TPR, 292 sensitivity) and small false positive rate (FPR, 1-specificity) based on the retention index 293 deviations of all identified compounds. For instance, the optimal similarity threshold for the 294 mixture of compound standards is 0.81 using ROC curve, where $\mathrm{TPR}=0.81$ and $\mathrm{FPR}=0.57$ 295 shown in the Figure 3.

296 As shown in Figure 2(b), retention indices of compounds identified using the optimal 297 similarity threshold of 0.81 are very close to the regression curve (the solid line). The suspected 298 compound in the dotted circle in Figure 2(a) was removed with the suggested optimal spectral 299 similarity threshold in the Figure 2(b). However, two compounds in the solid red circles in the 300 Figure 2(b) are still questionable. It is up to the users to decide whether these two compounds are 301 false identifications. It should be noted that the optimal threshold of spectrum similarity 302 recommended by EIder tends to be conservative since the method to find the optimal similarity 303 threshold using ROC curve is balance between TPR and FPR evenly. A practical threshold can 304 be set to a value lower than the recommended value in order to increase TPR, that is, to obtain 305 more true compounds in spite of high false identification. 
To further study the performance of EIder in analyzing complex samples, we derivatized a metabolite extract of mouse liver by MTBSTFA and analyzed the derivatized sample on GC×GC-TOF MS. A total of 1984 chromatographic peaks were detected by ChromaTOF. By setting threshold of mass spectral similarity to $\geq 0.6$, EIder identified 198 compounds corresponding to 281 chromatographic peaks using weighted cosine similarity measure. The optimal threshold of mass spectral similarity recommended by EIder was 0.76. By using this EIder recommended threshold, 114 compounds were identified from 138 chromatographic peaks using weighted cosine similarity measure.

Figure S9 depicts the relationship between the number of identified compounds and the threshold of mass spectrum similarity, ranging from 0.6 to 0.95 . It can be seen that an increase of mass spectrum similarity threshold decreases the numbers of derivatized, underivatized, as well as total compounds identified by mass spectrum matching. It is interesting to note that the number of identified compounds has a strong linear relationship with similarity threshold $\left(R^{2}>0.97\right)$

Table 2 shows the result of filtering compounds identified with the false derivatization reagent. A total of 8 MSTFA derivatized compounds were originally identified by mass spectrum matching. These identification results are false identifications because MTBSTFA was used as 324 the derivatization reagent during experiment. Therefore, EIder automatically removed these 325 compounds from the identification list based on the user provided derivatization information. By 326 doing so, 3 chromatographic peaks were identified as MTBSTFA-derivatized compounds. EIder

327 further automatically added the names and CAS numbers of the corresponding underivatized 328 compounds using our in-house created database, i.e., "4,6-Dimethyl-2-thioxo-1,2-dihydro-3329 pyridinecarbonitrile tbdms", "2-(aminooxy)acetic acid", and "L-Glutamic acid". To our 
330 knowledge, EIder is the only software package that has these derivatiztion-based filtering and 331 converting processes.

\section{3.3. Analysis of multiple samples from mouse feces}

333 It is a common practice in metabolomics that multiple samples are analyzed in a project to

334 increase its statistical power. Metabolite extracts from 25 mouse fecal samples were analyzed in 335 this study on a GC×GC-TOF MS system. The instrument data were first processed using 336 LECO's ChromaTOF software for peak picking. All peak lists generated from the 25 samples 337 were then aligned together by minimizing the retention time variation and maximizing pairwise 338 spectral similarity scores. A total of 99 peaks were fully aligned from the 25 peak lists; i.e., each 339 of these 99 peaks was detected and aligned together in all 25 samples. Supplementary 340 Information Figure S10 depicts the boxplot of relative standard deviation (RSD, \%) of the first 341 and the second dimension retention times. As expected, the second dimension retention has 342 larger relative standard deviation than the first dimension retention time.[13]

343 To identify compounds from GC-MS data, every mass spectrum acquired from each sample 344 is usually used as a query spectrum to find the best matched mass spectrum from a reference 345 library. The compound of the best matched spectrum is then considered as the compound giving 346 rise to the experimental mass spectrum. We termed this as the conventional approach. To reduce 347 the variations among the mass spectra of the same compound acquired in multiple samples, we 348 implemented an average mass spectrum approach in EIder software.

349 To calculate an average mass spectrum for a group of aligned mass spectra, each mass 350 spectrum is first normalized by setting the peak height of base peak to 1000 and then scaling the 351 peak height of the remaining peaks accordingly to form a normalized mass spectrum. The peak 352 heights of the same fragment ion in all normalized spectra are then added, and any fragment ions 
353 present in less than $75 \%$ of the normalized mass spectra are removed from the summed mass 354 spectrum as random peaks. The average mass spectrum is then calculated by dividing the peak 355 height of every fragment ion in the summed mass spectrum by the number of mass spectra.

356 Figure 4 depicts the performance comparison of the average spectrum approach and the 357 conventional approach in identification of compounds from the 99 fully aligned peaks. The mean 358 of spectral similarity scores calculated using the average spectrum approach and conventional 359 approach are 0.885 and 0.883 , respectively. In addition, the spectrum similarity scores of the 360 average spectrum approach are much tighter and have fewer outliers. Therefore, the proposed 361 average spectrum approach not only generates larger spectral similarity scores, but also improves 362 the distribution of the spectrum similarity scores.

Figure 5 shows an example that the conventional approach identified three compounds from 364 the mass spectra of a compound that were fully aligned in all 25 samples. Compound Malic acid, $3653 T B D M S$ derivative was identified from 14 mass spectra, while compound tert366 butyl(dimethyl)silyl 2-([tert-butyl(dimethyl)silyl]oxy)-3-methylbut-2-enoate was identified from 36710 mass spectra and compound 2-Pentamethyldisilanyloxybutane was identified from one mass 368 spectrum. The first two compounds are MTBSTFA derivatized. Therefore, EIder cannot filter 369 any of these three compounds based on either elementary composition or derivatization reagent. 370 However, the average mass spectrum approach identifies the compound giving rise to the 25 371 aligned peaks as Malic acid, 3TBDMS derivative, with a spectral similarity score of 0.662 . 372 Supplementary Information Figure S11 (a) and (b) are the average mass spectrum of the 25 373 aligned peaks and the reference mass spectrum in NIST 2011, respectively. The heaviest 374 fragment ion detected in each of the 25 mass spectra is $\mathrm{m} / \mathrm{z}=419$. The molecular weights of 375 Malic acid, 3TBDMS and tert-butyl(dimethyl)silyl 2-([tert-butyl(dimethyl)silyl]oxy)-3-methylbut- 
2-enoate are 477 and 343, respectively. It is impossible that the compound tertbutyl(dimethyl)silyl 2-([tert-butyl(dimethyl)silyl]oxy)-3-methylbut-2-enoate can generate a

378 fragment ion with $\mathrm{m} / \mathrm{z}=419$. For this reason, Malic acid, $3 T B D M S$ should be the true compound. 379 In fact, Figure 5 also shows that Malic acid, 3TBDMS has a large mean of spectrum similarity 380 scores and a tighter distribution than compound tert-butyl(dimethyl)silyl 2-([tert381 butyl(dimethyl)silyl]oxy)-3-methylbut-2-enoate. This demonstrates that the cross sample 382 alignment method implemented in EIder can correctly align the chromatographic peaks even 383 though they may have different identifications. Furthermore, the proposed average mass 384 spectrum approach in EIder is more robust for compound identification than the conventional 385 approach in a study that uses multiple samples.

\section{Conclusions}

While multiple compound identification algorithms have been reported in the literature and several commercial software packages are available, there is no open software available in the public domain for compound identification in GC-MS data. The developed EIder is an open compound identification software package. The EIder package provides eight literature reported spectral similarity measures for comprehensive identification analysis of EI spectra acquired on GC-MS or GC×GC-TOF MS. Two public open databases, WebBook and MassBank, are used as 393 its default reference mass spectrum library. The users have not only the option to add 394 commercial reference library such as NIST library, but also to build in-house reference libraries. 395 Multiple graphic user interfaces have been implemented in EIder to enable users to control false identification discovery using experimental information at different stages of analysis. EIder also

397 automatically links each identified compound to external websites to allow users to investigate detailed compound information. 


\section{Acknowledgements}

The authors thank Mrs. Marion McClain for review of this manuscript and the reviewers for their constructive suggestions. This work was supported by National Science Foundation (NSF) Grant DMS-1312603; National Institute of Health (NIH) Grants 1U01AA021910, 1U01AA021893, 1U01AA022489-01A1, and 1R01AA023681; the Veterans Administration; and the UofL Clinical and Translational Pilot Program.

\section{References}

[1] S.E. Stein, D.R. Scott, Optimization and testing of mass-spectral library search algorithms for compound identification, J. Am. Soc. Mass Spectr. 5 (1994) 859-866.

[2] X.L. Wei, I. Koo, S. Kim, X. Zhang, Compound identification in GC-MS by simultaneously evaluating the mass spectrum and retention index, Analyst 139 (2014) 2507-2514.

[3] G.T. Rasmussen, T.L. Isenhour, J.C. Marshall, Mass-spectral library searches using ion series data compression, J. Chem. Inf. Comp. Sci. 19 (1979) 98-104.

[4] I. Koo, X. Zhang, S. Kim, Wavelet- and Fourier-transform-based spectrum similarity approaches to compound identification in gas chromatography/mass spectrometry, Anal. Chem. 83 (2011) 5631-5638. [5] S. Kim, I. Koo, X.L. Wei, X. Zhang, A method of finding optimal weight factors for compound identification in gas chromatography-mass spectrometry, Bioinformatics 28 (2012) 1158-1163. [6] S. Kim, I. Koo, J. Jeong, S.W. Wu, X. Shi, X. Zhang, Compound identification using partial and semipartial correlations for gas chromatography-mass spectrometry data, Anal. Chem. 84 (2012) 64776487.

[7] I. Koo, S. Kim, X. Zhang, Comparative analysis of mass spectral matching-based compound identification in gas chromatography-mass spectrometry, J. Chromatogr. A 1298 (2013) 132-138.

[8] H. Horai, M. Arita, S. Kanaya, Y. Nihei, T. Ikeda, K. Suwa, Y. Ojima, K. Tanaka, S. Tanaka, K. Aoshima, Y. Oda, Y. Kakazu, M. Kusano, T. Tohge, F. Matsuda, Y. Sawada, M.Y. Hirai, H. Nakanishi, K. Ikeda, N.

Akimoto, T. Maoka, H. Takahashi, T. Ara, N. Sakurai, H. Suzuki, D. Shibata, S. Neumann, T. lida, K. Tanaka, K. Funatsu, F. Matsuura, T. Soga, R. Taguchi, K. Saito, T. Nishioka, MassBank: a public repository for sharing mass spectral data for life sciences, J. Mass Spectrom. 45 (2010) 703-714.

[9] S.E. Stein, Mass spectra, NIST Chemistry WebBook, NIST Standard Reference Database Number 69, Eds. P.J. Linstrom and W.G. Mallard, National Institute of Standards and Technology, Gaithersburg MD, 20899, http://webbook.nist.gov (retrieved April 15, 2013).

[10] I. Koo, X. Shi, S. Kim, X. Zhang, iMatch2: compound identification using retention index for analysis of gas chromatography-mass spectrometry data, J. Chromatogr. A 1337 (2014) 202-210.

[11] W. Liu, S. Lin, W.W. Piegorsch, Construction of exact simultaneous confidence bands for a simple linear regression model, Int. Stat. Rev. 76 (2008) 39-57. 
434 [12] J.A. Hanley, B.J. Mcneil, The meaning and use of the area under a receiver pperating characteristic 435 (Roc) curve, Radiology 143 (1982) 29-36.

436 [13] J.H. Winnike, X.L. Wei, K.J. Knagge, S.D. Colman, S.G. Gregory, X. Zhang, Comparison of GC-MS and 437 GCxGC-MS in the analysis of human serum samples for biomarker discovery, J. Proteome Res. 14 (2015) $438 \quad 1810-1817$. 
440 Table 1. Derivatization-based filtering results for analysis of compound standards. The top hit was

441 considered as the identification results. The Before Filtering columns show that MTBSTFA-derivatized

442 compounds were identified for the five chromatographic peaks by mass spectrum matching. The After

443 Filtering columns show the identification results by removing the MTBSTFA-derivitized compounds

444 from the identification list.

\begin{tabular}{|c|c|c|c|c|}
\hline \multirow[t]{2}{*}{$t_{R}(\min )$} & \multicolumn{2}{|l|}{ Before Filtering } & \multicolumn{2}{|l|}{ After Filtering } \\
\hline & Name & $\mathrm{SC}^{*}$ & Name & $\mathrm{SC}^{*}$ \\
\hline 26.65 & $\begin{array}{l}\text { L-Valine, N-(tert-butyldimethylsilyl)-, tert- } \\
\text { butyldimethylsilyl ester }\end{array}$ & 0.621 & $\begin{array}{l}\text { L-Proline, 1-(trimethylsilyl)-, trimethylsilyl } \\
\text { ester }\end{array}$ & 0.612 \\
\hline 26.69 & $\begin{array}{l}\text { Glycine, N-(tert-butyldimethylsilyl)-, tert- } \\
\text { butyldimethylsilyl ester }\end{array}$ & 0.656 & Glycine, N,N-bis(trimethylsilyl)-, methyl ester & 0.618 \\
\hline 28.89 & $\begin{array}{l}\text { Glycine, N-(tert-butyldimethylsilyl)-, tert- } \\
\text { butyldimethylsilyl ester }\end{array}$ & 0.620 & $\begin{array}{l}\text { Pentanoic acid, 4-methyl-2- } \\
\text { [(trimethylsilyl)oxy]-, trimethylsilyl ester }\end{array}$ & 0.589 \\
\hline 31.02 & $\begin{array}{l}\text { Butanoic acid, 4-[(tert- } \\
\text { butyldimethylsilyl)amino]-, tert- } \\
\text { butyldimethylsilyl ester }\end{array}$ & 0.695 & Androstan-17-one, (5à)- & 0.595 \\
\hline 34.74 & $\begin{array}{l}\text { Decanedioic acid, bis(tert-butyldimethylsilyl) } \\
\text { ester }\end{array}$ & 0.634 & Benzene, eicosyl- & 0.582 \\
\hline
\end{tabular}

$445 *$ weighted cosine similarity score 
447 Table 2. List of compounds identified from mass spectra of eight chromatographic peaks with and 448 without applying derivatization-based filtering. The threshold of similarity score was set as 0.8 .

\begin{tabular}{|c|c|c|}
\hline$t_{R}(\min )$ & Before filtering derivatized approach & After filtering derivatized approach \\
\hline 13.53 & $\begin{array}{l}\text { Ethanamine, N,N-dimethyl-2- } \\
\text { [(trimethylsilyl)oxy]- }\end{array}$ & $\begin{array}{l}\text { 2-Amino-2-methyl-1-propanol, } \\
\text { Isobutanolamin }\end{array}$ \\
\hline 17.33 & $\begin{array}{l}\text { Propanoic acid, 2-(methoxyimino)-, } \\
\text { trimethylsilyl ester }\end{array}$ & $\begin{array}{l}\text { 1-Norvaline, N-(2-methoxyethoxycarbonyl)-, } \\
\text { octyl ester }\end{array}$ \\
\hline 19.13 & Tris(trimethylsilyl)borate & $\begin{array}{l}\text { 4,6-Dimethyl-2-thioxo-1,2-dihydro-3- } \\
\text { pyridinecarbonitrile tbdms }\end{array}$ \\
\hline 25.10 & Tris(trimethylsilyl)carbamate & 2-(aminooxy)acetic acid \\
\hline 25.33 & Ethanedioic acid, bis(trimethylsilyl) ester & 1-Pentamethyldisilyloxy-10-undecene \\
\hline 28.20 & $\begin{array}{l}\text { 1-(2-Methoxyethoxy)-2-methyl-2-propanol, } \\
\text { trimethylsilyl ether }\end{array}$ & Silane, butoxytrimethyl- \\
\hline 39.77 & $\begin{array}{l}\text { L-Glutamic acid, N-(tert- } \\
\text { butyldimethylsilyl)-, bis(tert- } \\
\text { butyldimethylsilyl) ester }\end{array}$ & L-Glutamic acid \\
\hline 46.13 & Arachidonic acid, trimethylsilyl ester & $\begin{array}{l}\text { 4,7,10,13,16,19-Docosahexaenoic acid, } \\
\text { methyl ester, (all-Z)- }\end{array}$ \\
\hline
\end{tabular}


452 Figure 1. Workflow of compound identification in software EIder.

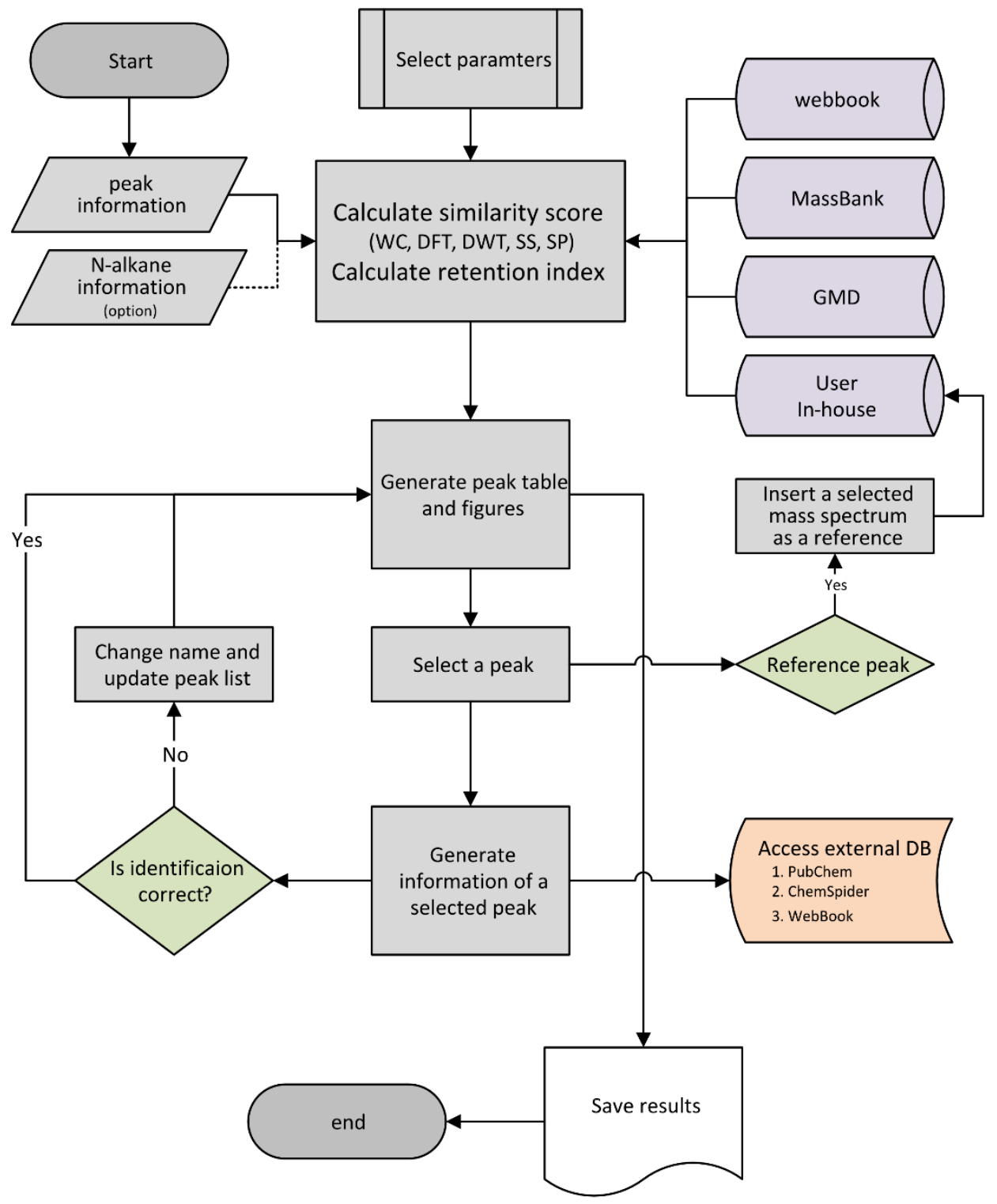


455 Figure 2. Auxiliary figure of retention index versus retention time corresponding to threshold of

456 similarity scores 0.6 in (a) and 0.81 in (b). The dotted and solid circles indicate compounds that are

457 outside of $95 \%$ confidence bands in regression analysis.

(a)

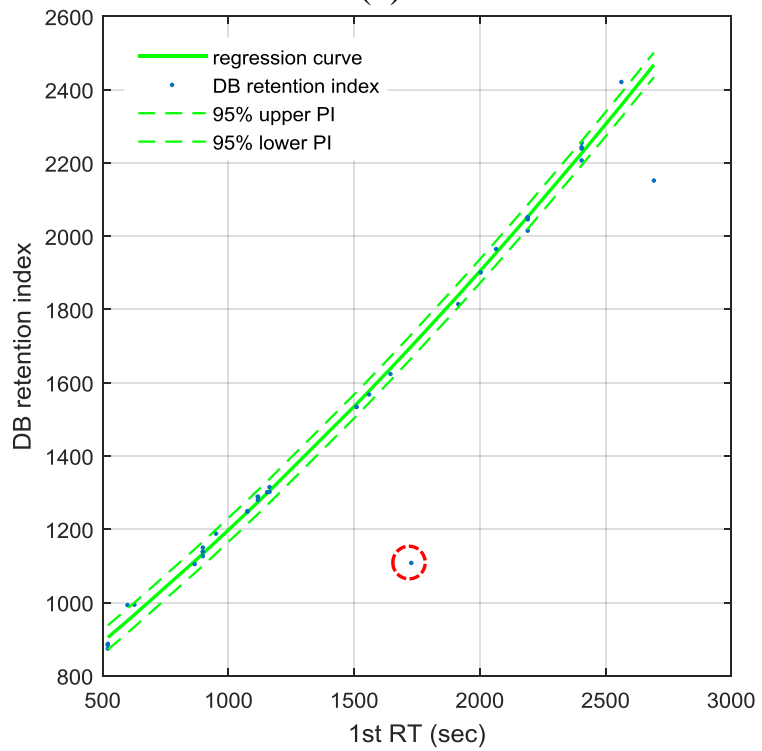

(b)

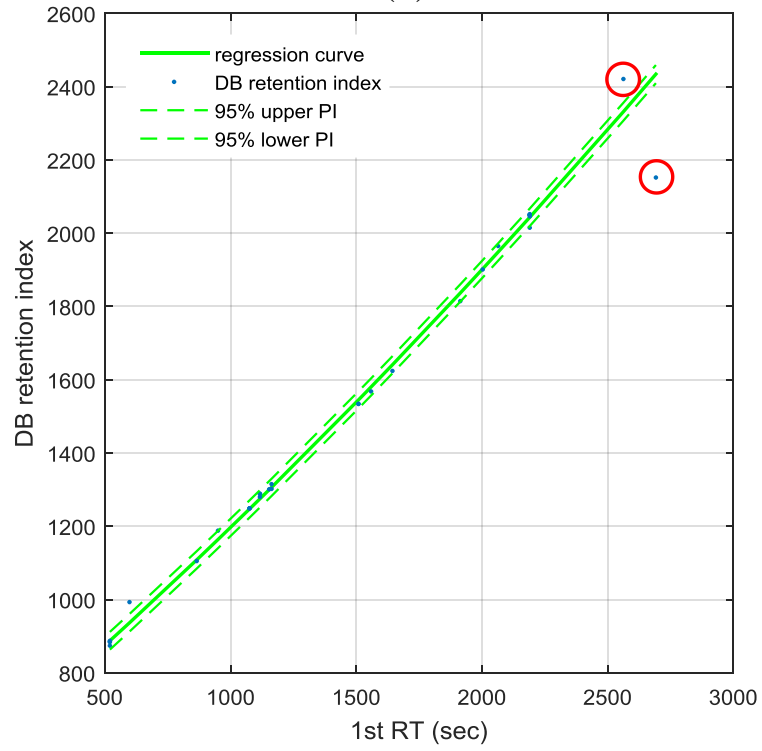

458 
460 Figure 3. Receiver operating characteristic (ROC) curve and the EIder recommended optimal threshold 461 of similarity score. The red circle represents estimated optimal threshold, where the suggested optimal 462 similarity threshold is 0.81 with TPR (sensitivity) $=0.81$ and FPR (1-specificity) $=0.57$.

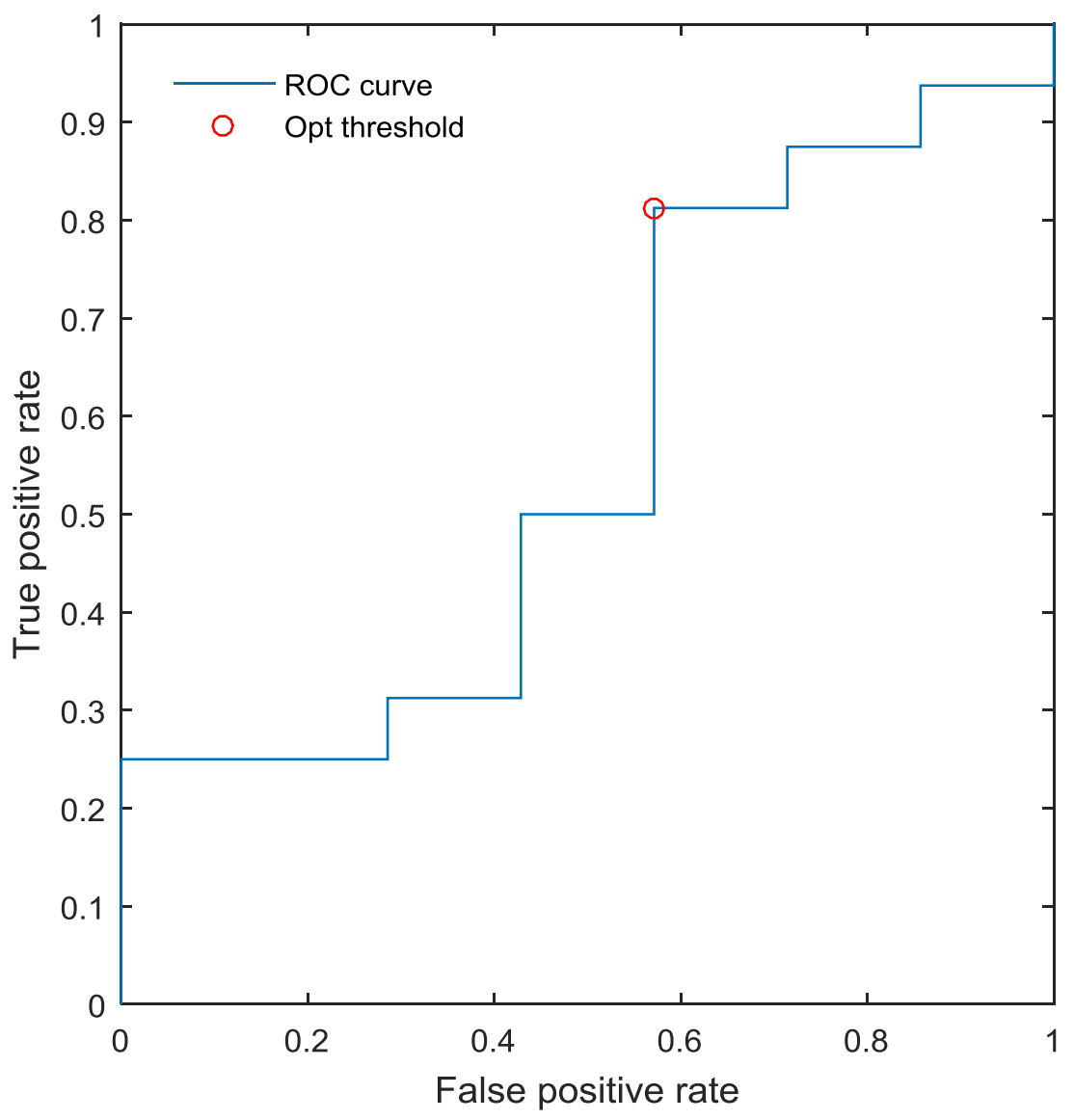

464

465 
466 Figure 4. Boxplot of spectral similarity scores of the 99 fully aligned compounds calculated using 467 average mass spectrum approach and conventional pairwise comparison.

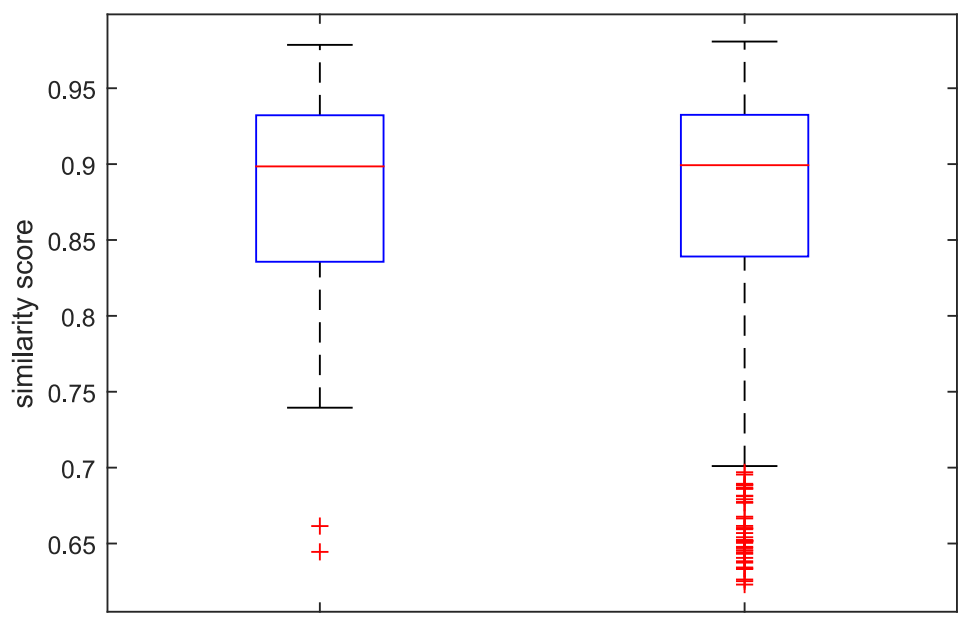

average mass spectra

conventional

470 Figure 5. Boxplot of similarity scores of each unique compound name in the example aligned peak.

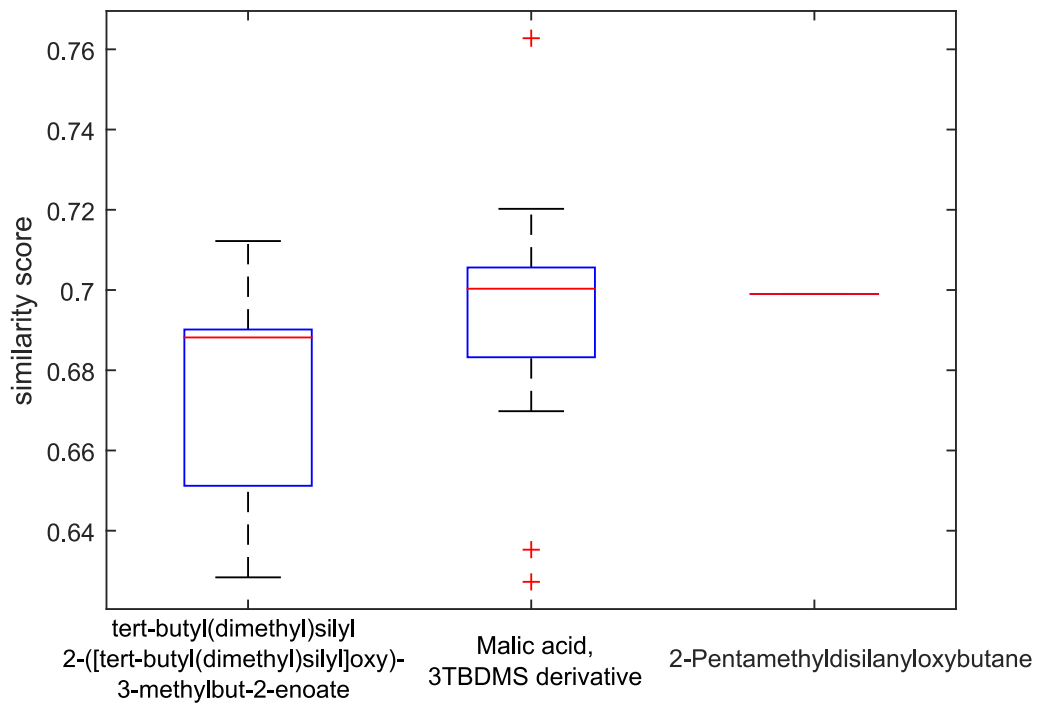

